\title{
The ins and outs of cells
}

\author{
The sensors and switches that convert environmental inputs to specific biological outputs inspire the tools \\ chemical biologists engineer and apply to better understand these complex systems.
}

Cells must integrate and translate a barrage of inputs into specific biological outputs. After inputs are detected by biological sensors, the flow of information to outputs depends on switches, much like the flow of electrons in a circuit. Because a myriad of factors can influence a cell and the downstream responses are similarly variable, sensors and switches themselves are diverse and complex. In this focus issue, we examine the details of specific molecules that are emerging as important biological sensors or switches and also revisit some well-precedented systems where the remaining challenges are not in obtaining a molecular-level understanding of individual components, but in deciphering the integration of switch circuitry. Other contributions to this special issue highlight the tools inspired by nature's sensors and switches as well as new insights and questions that their applications have uncovered.

A biological sensor can be defined simply by its ability to detect and report on an input, whether internal or external in origin. Sensors rely on several mechanisms to demonstrate that a signal has been received, many of which involve a reversible structural change. In particular, Gill and colleagues (Commentary, p. 488) describe how the dissociation of calcium from STIM proteins induces a conformational change that exposes a protein-protein interaction domain, thus reporting on calcium depletion in the endoplasmic reticulum. Carling and colleagues (Perspective, p. 512) describe new insights into how adenine nucleotides affect allosteric control over AMP-activated protein kinase (AMPK), a key regulator of energy metabolism in eukaryotic cells.

Sensing and switching activity can be consolidated in the same molecule in cases where detection causes a change in enzyme activity or in subcellular localization. In their article on AMPK, Carling and colleagues (p. 512) point out that binding of adenine nucleotides preserves enzyme activity by preventing dephosphorylation. Cherfils and Zeghouf (Commentary, p. 493)

discuss the relocalization of GTPases, which is coupled to the exchange of guanine cofactors. Detection can also be read out as a physical change to the sensor: when directly altered by chemically reactive molecules, the modifications themselves can serve as switches. In this regard, Dickinson and Chang (Perspective, p. 504) discuss the localized production of reactive oxygen species (ROS) in cells as well as the biomolecules that are sensitive to these species, making it clear that these modifications can impact protein activity. Identifying these modifications and explaining their functional role, however, remains a challenge.

As illustrated above, cellular inputs are often metals or small molecules; nucleotides are somewhat privileged inputs, because hydrolysis of high-energy phosphate bonds can power downstream switches. However, inputs can also include complex biomolecules, light or temperature, and sensors often detect several inputs to offer a more nuanced or robust cellular response. For example, in their discussion of STIM proteins, Gill and colleagues (p. 488) offer the progressive view that these proteins respond to multiple inputs of cellular stress, including fluctuations in calcium, ROS or temperature. Carling and colleagues (p. 512) also touch on this theme, highlighting AMPK's responsiveness to changes in intracellular calcium or possibly glycogen.

These articles illustrate the complexity of cellular inputs, yet cellular responses can be similarly convoluted. For example, outputs can be transient, occurring rapidly but ending quickly; long-lived, resulting in the formation of an alternative stable state; or dynamic, producing an oscillating or cyclic outcome. The circuits that allow for complex output options often incorporate multiple feedback and feed-forward loops. Ptashne (Commentary, p. 484) uses the well-characterized transcriptional switch from bacteriophage lambda to illustrate the robustness of complex circuits, exploring the components that make them respond quickly to a specific input yet keep them relatively insensitive to small perturbations in the circuit itself. Indeed, Ptashne raises the possibility that all switches are governed by similar rules, perhaps implying that switches that appear simple may be ripe for further investigation. This appears to be true in quorum sensing, where - as Jung reports - assigning different functions to multiple feedback loops in the context of complex input-output relationships is particularly difficult (News \& Views, p. 502). Finally, circuit networks can yield unexpected outcomes, in some cases with the same molecular components producing divergent cellular responses. Cherfils and Zeghouf (p. 493) highlight the challenge of understanding how GTPase switches work when the inputs they receive and the outputs they control far outnumber the GTPases themselves. Similarly, G proteincoupled receptors continue to astound, as unprecedented insights into output choice are emerging from relating newly available structures to functional data about ligand activation.

Despite the challenges in realizing a comprehensive systems-wide view of biological switches, efforts toward obtaining a detailed understanding of the components that detect inputs and govern outputs have inspired chemical biology. For example, Fussenegger and colleagues (Research Highlights, p. 497) have taken advantage of the photoswitch in melanopsin to engineer a system allowing quantitative control over protein expression in vivo. Lemke and Schultz (Commentary, p. 480) describe their view of the types of biomolecules most suitable for adaptation as synthetic sensors and provide recommendations for how to engineer tools best suited to particular applications. Famulok and colleagues (p. 519) review the design of aptamers, based largely on natural riboswitches, for allosteric control of gene expression. This review also issues a call to the synthetic biology community for additional aptamer-ligand pairs that can be applied to new biological space or used in combinations, yielding more sophisticated control over a system. Similarly, Dickinson and Chang (p. 504) challenge the ROS community to engineer tools that can distinguish individual ROS based on their unique chemistries, so the emerging view of these molecules as bona fide biological signals can be more fully explored.

Although biological switches have been studied for decades, this continues to be a rich and rewarding field of investigation. Like the systems that they study, chemical biologists contribute to and benefit from a feed-forward research cycle whereby new molecular insights enable the development of more sophisticated tools that, in turn, allow scientists who use them to tackle increasingly complex questions. We look forward to the next loop of this cycle. 